\title{
Residência Multiprofissional em Saúde da Família e a Formação de Psicólogos para a Atuação na Atenção Básica
}

\section{Family Health Multiprofessional Residency and the Education of Psychologists to Work in Basic Care}

\author{
Anselmo Clemente \\ Psicólogo, Caps/ Prefeitura de Itanhaém-SP. \\ Endereço: Rua Urcesino Ferreira, 690, Bairro Baixio, CEP 117 40-000, \\ Itanhaém, São Paulo, SP, Brasil. \\ E-mail: anselmo.euœbol.com.br

\section{Damaris Roma Matos} \\ Especialista em Saúde da Família. Psicóloga, Faculdade Santa Mar- \\ celina. \\ Endereço: Rua Miguel Langone, 94 apto 6I, Itaquera, CEP 08215-330, \\ São Paulo, SP, Brasil. \\ E-mail: damarismatos®itelefonica.com.br \\ Danitielle K. Marques Grejanin \\ Psicóloga Especialista em Saúde da Família, Gestora Local, Casa de \\ Saúde Santa Marcelina. \\ Endereço: Rua Arraial da Anta, 302 apto II3, Itaquera, CEP 08270- \\ 090, São Paulo, SP, Brasil. \\ E-mail: danitimarquesळyahoo.com.br

\section{Heloísa Elaine dos Santos} \\ Psicóloga Especialista em Saúde da Família e em Psicologia Hospi- \\ talar. Secretaria da Saúde do Município de Diadema/SP. Discente \\ do Programa de Residência Multiprofissional em Saúde da Família \\ da Faculdade Santa Marcelina. \\ Endereço: Rua Mario Calazans Machado, 144, CEP 03589-070, São \\ Paulo, SP, Brasil. \\ E-mail: heloisa_elaine_santosळyahoo.com.br

\section{Michele Peixoto Quevedo} \\ Mestre em Saúde Pública. Psicóloga da Coordenação do Programa \\ de Saúde da Família/Supervisor técnico da Residência Multi- \\ profissional em Saúde da Família - categoria Psicologia Casa de \\ Saúde Santa Marcelina. \\ Endereço: Rua Fontoura Xavier 1037, Vila Carmosina, Itaquera, CEP \\ 08295-300, São Paulo, SP, Brasil. \\ E-mail: mquevळhotmail.com \\ Paula Andrea Massa \\ Especialista em Saúde da Família. Psicóloga e Técnica em mediação \\ de conflito. Associação Novolhar e Pró-Mulher família e Cidadania. \\ Endereço: Av. Liberdade, 32, CEP 01502-000, São Paulo, SP, Brasil. \\ E-mail:paula_psicoooळyahoo.com.br
}

\section{Resumo}

O Programa de Saúde da Família (PSF) surgiu como estratégia de reorientação da atenção básica em saúde. A incorporação de profissionais de saúde mental no PSF não foi prevista na Portaria nº 648 (2006), que definiu os recursos mínimos para a atuação das equipes. O Ministério da Saúde, em 2007, aprovou os Núcleos de Atenção à Saúde da Família. A Residência Multiprofissional em Saúde da Família foi uma proposta coordenada pela Faculdade e Casa de Saúde Santa Marcelina, contando com dez categorias profissionais, entre elas a psicologia. Este trabalho tem o objetivo de refletir sobre a prática do psicólogo residente e analisá-la, contribuindo para a discussão das possibilidades de atuação do psicólogo no PSF na cidade de São Paulo. Trata-se de uma pesquisa avaliativa do tipo estudo de caso, que tem o objetivo de sistematizar as intervenções realizadas, o processo de construção da planilha mensal de produção e alguns dados que ela fornece. Os resultados são: a descrição das categorias, a planilha mensal de produção com adaptações a partir dos códigos do Sistema de Informação Ambulatorial (SIA) atribuídos aos psicólogos e um quadro com os dados quantitativos. Foi possível perceber que os princípios do PSF, a formação em psicologia e a vinculação com a Residência estão relacionados com os caminhos percorridos. Concluiu-se, então, que a quantificação é necessária para a visualização das ações, entretanto, não é suficiente para avaliar a abrangência, a eficácia e a efetividade das ações e o quanto o SIA não está preparado para o registro, o controle e a fiscalização do psicólogo inserido no PSF.

Palavras-chave: Avaliação em saúde; Programa de Saúde da Família; Psicologia. 


\section{Abstract}

The Family Health Program (FHP) emerged in Brazil as a strategy of basic health care reorientation. The incorporation of Mental Health professionals in the FHP was not included in Directive 648 (year?), which defines minimum resources for the teams' work. In 2007, the Brazilian Ministry of Health approved the Family Healthcare Nuclei. The Multiprofessional Family Health Residency was a proposal that was coordinated by the Santa Marcelina Faculty and Hospital. It had ten professional categories, one of them being the psychologists. This work aims to reflect on and analyze the resident psychologists' practice and contribute to the discussion about the possibilities for the psychologists' work in the FHP in the city of São Paulo. It is a descriptive research; more specifically, a case study. The objective is the systematization of the practices, of the process of construction of the monthly production table and of some data it provides. The results are: categories description, the monthly production table, with some adaptations based on the codes of the Day-Care Information System that were attributed to psychologists, and a framework with quantitative data. It was possible to notice that: FHP principles, having attended a psychology undergraduation course and being part of the residency program are related with the psychologists' own history. The conclusion is that, although quantifying is necessary for the understanding of the practices, it is not sufficient to assess the complexity, efficacy and effectiveness of the practices. In addition, quantifying does not reveal the extent to which the DayCare Information System is not prepared to register, control and inspect the psychologists who work in the FHP.

Keywords: Health Evaluation; Family Health Program; Psychology.

\section{O PSF como Estratégia da Atenção Básica}

Desde 1994, o Programa de Saúde da Família (PSF) vem se configurando como principal estratégia reorganizadora da atenção básica do Sistema Único de Saúde (SUS), sua expansão e consolidação são tendências em todo país. Seus princípios fundamentais são a integralidade, a qualidade, a eqüidade e a participação social. A partir do cadastro dos usuários, as Equipes Saúde da Família (ESF) vinculam-se à população, possibilitando o compromisso e a co-responsabilidade desses profissionais com os usuários e a comunidade. (Brasil, 2007a)

Para Camargo-Borges e Cardoso (2005), os objetivos do PSF estão relacionados ao atendimento das minorias sem acesso a serviços de saúde, como resposta a uma tendência mundial de redução de custos, além da humanização dos serviços, promovendo uma desmedicalização da medicina. Segundo Capistrano Filho (1999), o PSF foi implantado, inicialmente, em cidades de pequeno porte com escassez de serviços e profissionais.

Apesar do relativo sucesso que o programa obteve nessas localidades, ao analisar a evolução da cobertura do PSF, nos anos de 1998 a 2003, em grandes centros urbanos, Viana (2006) concluiu que, em contraste com a maior disponibilidade de oferta de serviços de saúde (com ênfase para atenção de média e alta complexidade), esses municípios de grande porte apresentaram "limitações quanto à qualidade e capacidade de resposta dos serviços” (Viana, 20o6, p. 579) e ritmo mais lento de expansão de cobertura da população cadastrada.

Na cidade de São Paulo, a Secretaria Municipal de Saúde tem utilizado algumas estratégias que visam à organização e à qualidade da atenção básica, dentre elas, os Documentos Norteadores de 2002 e 2005 pactuados no consenso entre interlocutores regionais, coordenadores distritais e assessores do PSF. $\mathrm{O}$ documento publicado em 2002 define as atribuições profissionais específicas para a estratégia de saúde da família; o de 2005, norteia o monitoramento da atenção básica por meio de indicadores oficiais próprios (São Paulo, 2007a; 2007b). 


\section{PSF e Saúde Mental}

A municipalização da atenção básica aconteceu a partir de 2001, com a manutenção e a expansão do modelo de parcerias no PSF. Segundo Alves Sobrinho e Capucci (2003), em 2001, existiam 12 parceiras administrando o PSF na grande São Paulo por meio da incorporação ativa da rede formada por organizações sociais que, com relativo grau de autonomia, podiam ou não incluir equipes de saúde mental com a presença de profissionais de psicologia.

Segundo levantamento do Departamento de Atenção Básica do Ministério da Saúde, "as equipes se deparam, cotidianamente, com problemas de 'saúde mental': $56 \%$ das equipes de saúde da família referiram realizar 'alguma ação de saúde mental'” (Brasil, 20o6b, p. 3). Destaca-se, a importância da integração entre a assistência prestada em saúde mental e a atenção básica.

No entanto, a Política Nacional de Atenção Básica, por meio da Portaria $\mathrm{n}^{\circ} .648$, em 2006, definiu os recursos mínimos necessários para a atuação das equipes de PSF, e não previu uma equipe de saúde mental na equipe de Saúde da Família. E, “embora os municípios e estados tenham autonomia de mudar a estrutura do PSF, não há orientação por parte do Ministério da Saúde para que sejam incluídas equipes de saúde mental" (CRP, 2006, p. 6).

Entretanto, em agosto de 2007, o Ministério da Saúde publicou a aprovação, na Comissão Intergestora Tripartite, dos Núcleos de Apoio a Saúde da Família (NASF), que estariam vinculados às equipes mínimas de PSF e compartilhariam as práticas de saúde nos territórios sob responsabilidade dessas equipes. Serão constituídos, de acordo com a necessidade local, por equipes de até 5 profissionais de ensino superior de diferentes áreas do conhecimento (inclusive o profissional de psicologia). Há orientação para que todas as equipes dos NASF tenham no mínimo um profissional da saúde mental (Brasil, 2007d).

\section{Residência Multiprofissional: formação em serviço - Saúde Mental e a Atenção Básica}

A Portaria Interministerial 2118, de o3 de novembro de 2005 , instituiu a parceria entre o Ministério da
Educação e o Ministério da Saúde para cooperação técnica e desenvolvimento de recursos humanos na área de saúde (Brasil, 2007b). Esta tem o intuito de atender à Constituição Federal de 1988, artigo 200, inciso III, que determina que "Ao SUS compete [...] ordenar a formação de recursos humanos na área de saúde" (Brasil, 2006a, p. 132).

No ano de 2005, o Ministério da Saúde financiava 22 programas de residência multiprofissional em funcionamento no Brasil, num total de 1558 residentes de diversas categorias. Esses programas estavam distribuídos em vários Estados do país e possuíam configuração variada. As categorias profissionais incluídas e a ênfase de cada programa obedecem às demandas locais e aos projetos das instituições formadoras. A psicologia foi incluída em vários deles, em especialidades como: saúde pública e saúde da família.

\section{Residência Multiprofissional da Faculdade e Casa de Saúde Santa Marcelina}

A primeira Residência Multiprofissional em Saúde da Família (2005-2007) com a participação de dez categorias profissionais distintas (enfermagem, medicina, odontologia, psicologia, fisioterapia, terapia ocupacional, fonoaudiologia, nutrição, serviço social e farmácia), totalizando em média 70 residentes, foi coordenada por um colegiado interinstitucional composto pela Casa de Saúde Santa Marcelina, o Núcleo de Capacitação em Saúde da Família e a Faculdade Santa Marcelina.

Os residentes foram inseridos em Unidades Básicas de Saúde (UBS) com PSF na Zona Leste de São Paulo, que foram previamente selecionadas a partir do perfil e da disponibilidade dos tutores e aceitação por parte da gerência e dos profissionais do serviço. Ocorreu, então, a divisão dos residentes em 16 grupos de 5 ou 6 componentes, formando equipes multiprofissionais que se atrelaram às equipes de Saúde da Família já existentes em 16 UBSs. 0 grupo de residentes contou com um tutor médico e um enfermeiro (responsáveis pelo acompanhamento em serviço) e com a preceptoria específica de sua categoria profissional (responsável pela operacionalização teórica para aplicação em serviço) (Bourget e col., 2006) 
As atividades realizadas pelos residentes foram organizadas de forma que $50 \%$ do período de trabalho fosse dedicado às atividades com a equipe multiprofissional e os outros $50 \%$ às atividades específicas de sua categoria. Também havia a participação: (a) diária, nas reuniões da equipe de saúde da família a que se reportavam, (b) quinzenais, nas supervisões de casos, (c) duas vezes por semana nas aulas teóricas (gerais e específicas) e (d) eventualmente, conforme a disponibilidade de cada residente e da realidade de cada área, nas reuniões do conselho gestor.

As UBSs que receberam os residentes de psicologia foram A. E. Carvalho, Dom Angélico, Dom João Nery, Jardim Fanganiello e Santa Inês. De forma geral, o objetivo de todas as ações era a promoção da saúde e a prevenção, além de ações curativas, preconizando o exercício do trabalho em equipe e da atenção voltada à família e não apenas ao indivíduo isolado de seu contexto sócio-familiar.

\section{Objetivo}

Este trabalho tem o objetivo de refletir e analisar a prática do psicólogo residente do Programa de Residência Multiprofissional em Saúde da Família da Faculdade e Casa de Saúde Santa Marcelina e contribuir para a discussão sobre as possibilidades de atuação do psicólogo no Programa de Saúde da Família da Atenção Básica da cidade de São Paulo.

\section{Método}

Trata-se de uma pesquisa avaliativa do tipo estudo de caso. A unidade-caso é a experiência dos residentes de psicologia no Programa de Saúde da Família e, mais especificamente, a sistematização das intervenções realizadas, o processo de construção da planilha mensal de produção e alguns dados que ela fornece já que não é o foco deste trabalho fazer uma apresentação da produção dos psicólogos residentes.

\section{Resultados}

Os psicólogos residentes realizaram por dois anos atividades multiprofissionais e específicas da categoria. As intervenções realizadas foram escolhidas de acordo com as prioridades do Programa de Saúde da
Família (diabéticos, hipertensos, gestantes, menores de um ano, etc.), a forma como ele se organiza, a expectativa e a demanda identificada pelos profissionais da equipe (residentes e tutoria), a demanda espontânea da população e, por fim, a busca ativa que os psicólogos residentes fizeram ao longo do processo.

Dessa forma, as queixas encontradas com maior freqüência foram os sintomas depressivos, estresse, ansiedade, ideação suicida, problemas de aprendizagem, alteração de comportamento, entre outros.

A sistematização das intervenções foi desenvolvida pela construção e descrição de categorias baseadas na prática dos residentes. As categorias e suas descrições são: consulta (todo primeiro contato para triagem ou pronto-atendimento), terapia (atendimentos realizados para psicodiagnóstico e psicoterapia individual para todas as faixas etárias e psicoterapia familiar), grupos (que podem ser 1. Terapêuticos: grupos com fins terapêuticos que visam à melhoria de uma situação de patologia dos indivíduos e que foram realizados, por exemplo, com as seguintes demandas, tabagistas, pacientes com diagnóstico de ansiedade, depressão, etc. ou 2. Educativos: grupos com objetivo de educar/capacitar tanto a comunidade quanto os profissionais da UBS, podem ser realizados por equipe multiprofissional e acontecem na unidade ou na comunidade), oficinas terapêuticas (encontros com fins terapêuticos e promoção da qualidade de vida, nos quais são utilizadas como metodologia: atividades lúdicas, ocupacionais e troca de experiências, podem ser tanto para a comunidade quanto para os profissionais da UBS); visita domiciliar (realizadas para orientação, triagem e/ou atendimento terapêutico. Também realizadas com outros profissionais da equipe).

A partir dessa sistematização, surgiu a necessidade de organizar os dados produzidos com o objetivo de traçar o perfil da população atendida (quantidade por faixa etária), bem como das atividades realizadas (tipos de atividades, quantidade e tempo gasto com cada). Para isso, foi elaborada uma planilha mensal de produção.

Após o primeiro esboço desse instrumento, houve a necessidade de consultar o Sistema de Informações Ambulatoriais (SIA) do SUS (Brasil, 2007c), que é uma "fonte de registro, controle e fiscalização de todos os serviços prestados pela rede assistencial" (Oliveira e col., 2005, p. 275). Para os profissionais da psicologia, existem 53 procedimentos codificados no SIA. 
Ao comparar a prática dos residentes com os procedimentos assistenciais preconizados por este sistema, foram escolhidos diversos códigos atribuídos aos psicólogos nos diferentes níveis de atenção: básica, média e alta complexidade. Os códigos escolhidos foram os que mais se aproximavam das categorias já descritas na sistematização supracitada, ainda assim, vale ressaltar que além de emprestar os códigos do SIA, houve a necessidade de uma adaptação conceitual deles à realidade vivida pelos residentes.
A planilha mensal de produção é dividida em duas tabelas para preenchimento diário. A primeira (Quadro 1) refere-se aos grupos de atividades nomeados a partir da sistematização feita pelos psicólogos residentes. É preenchida com o número de pessoas beneficiadas em cada grupo de atividade por faixa etária. Na segunda tabela (Quadro 2), na qual os códigos do SIA são utilizados e adaptados, os campos referem-se a subtipos de atividades pertencentes aos grupos da primeira tabela e em seguida, os procedimentos possíveis para os diversos subtipos.

\section{Quadro I - Planilha Mensal do número de pessoas atendidas em cada grupo de atividade por faixa etária (Folha I)}

\begin{tabular}{|c|c|c|c|c|}
\hline Grupos de Atividades & Faixa etária & \multicolumn{3}{|c|}{ Dias do mês } \\
\hline \multirow{6}{*}{ I. Consultas } & $0-4$ anos & & & \\
\hline & $5-12$ anos & & & \\
\hline & $13-21$ anos & & & \\
\hline & $22-59$ anos & & & \\
\hline & 60 anos ou mais & & & \\
\hline & Total & 0 & $\circ$ & 0 \\
\hline \multirow{6}{*}{ 2. Terapia } & $0-4$ anos & & & \\
\hline & $5-12$ anos & & & \\
\hline & $13-21$ anos & & & \\
\hline & $22-59$ anos & & & \\
\hline & 60 anos ou mais & & & \\
\hline & Total & $\circ$ & $\circ$ & 0 \\
\hline \multirow{6}{*}{ 3. Grupos } & $0-4$ anos & & & \\
\hline & $5-12$ anos & & & \\
\hline & $13-21$ anos & & & \\
\hline & $22-59$ anos & & & \\
\hline & 60 anos ou mais & & & \\
\hline & Total & 0 & 0 & 0 \\
\hline \multirow{6}{*}{ 4. Oficina terapêutica } & $0-4$ anos & & & \\
\hline & $5-12$ anos & & & \\
\hline & $13-21$ anos & & & \\
\hline & $22-59$ anos & & & \\
\hline & 60 anos ou mais & & & \\
\hline & Total & $\circ$ & $\circ$ & o \\
\hline \multirow{6}{*}{ 5. Visitas domiciliares } & $0-4$ anos & & & \\
\hline & $5-12$ anos & & & \\
\hline & $13-21$ anos & & & \\
\hline & $22-59$ anos & & & \\
\hline & 60 anos ou mais & & & \\
\hline & Total & 0 & ० & 0 \\
\hline
\end{tabular}


Quadro 2 - Planilha Mensal do número de ações realizadas por subtipo de atividade (Folha 2)

\begin{tabular}{|c|c|c|c|c|}
\hline & Subtipos de atividades & \multicolumn{3}{|c|}{ Dias do mês } \\
\hline $0401105-8$ & I A-Consulta individual/pronto-atendimento & & & \\
\hline $0401105-8$ & I B-Consulta individual/triagem & & & \\
\hline $070216-2$ & 2 A - Terapia individual & & & \\
\hline $0702105-4$ & 2 B -Terapia de grupo/família & & & \\
\hline $0702105-4$ & 3 A-Terapia de grupo & & & \\
\hline $0401102-3$ & 3B-Ativ. educativa/Capac. na comunidade & & & \\
\hline $0401103-1$ & $3 \mathrm{C}$-Ativ. educativa/Capac. na unidade & & & \\
\hline $4001201-8$ & 4 A-Reeducação/reinserção social & & & \\
\hline $1915107-1$ & 4B-Oficina terapêutica/Saúde mental & & & \\
\hline $4001401-0$ & 5 A-Visita domiciliar/Visita institucional & & & \\
\hline $0401107-4$ & 5 B - Visita domiciliar/Atendimento & & & \\
\hline $0401107-4$ & ${ }_{5} \mathrm{C}-$ Visita domiciliar/Multiprofissional & & & \\
\hline \multirow[t]{3}{*}{$0401107-4$} & 5D-Visita domiciliar/Orientação/Triagem & & & \\
\hline & Total & 0 & 0 & 0 \\
\hline & Procedimentos & & & \\
\hline $0702104-6$ & Aplicação de testes para psicodiagnóstico & & & \\
\hline 4001101-1 & Acompanhamento psicológico em reabilitação & & & \\
\hline $4001103-8$ & Acompanhamento neuropsicológico & & & \\
\hline $4001102-0$ & Acompanhamento neurodesenvolvimento & & & \\
\hline $4001104-6$ & Acompanhamento psicopedagógico em reabilitação & & & \\
\hline $1916101-8$ & Abordagem do fumante & & & \\
\hline $4001501-7$ & Orientação em reabilitação & & & \\
\hline \multirow[t]{8}{*}{$4001112-7$} & Acompanhamento multidisciplinar em reabilitação & & & \\
\hline & Total & 0 & 0 & 0 \\
\hline & Total de consultas no dia & & & \\
\hline & Número de pacientes agendados (A) & & & \\
\hline & Número de pacientes eventuais/orientações (B) & & & \\
\hline & Número de faltas às consultas $\quad$ (C) & & & \\
\hline & Número de pacientes desmarcados (D) & & & \\
\hline & Número total de consultas no dia $(A+B-C-D)$ & 0 & 0 & 0 \\
\hline & Distribuição da carga horária & & & \\
\hline \multirow{13}{*}{$\begin{array}{l}\text { Períodos } \\
\text { trabalhados } \\
\text { (em horas) }\end{array}$} & Consultas/Atendimentos & & & \\
\hline & Grupos/Oficinas & & & \\
\hline & Reuniões de equipe & & & \\
\hline & Atividades educativas & & & \\
\hline & Visitas domiciliares/institucionais & & & \\
\hline & Cursos/congressos & & & \\
\hline & Trabalhos com equipe multiprofissional & & & \\
\hline & Trabalhos administrativos & & & \\
\hline & Práticas acadêmicas & & & \\
\hline & outros; & & & \\
\hline & Subtotal & 0,0 & 0,0 & 0,0 \\
\hline & Férias/licença/abonos/faltas & & & \\
\hline & Total & 0,0 & 0,0 & 0,0 \\
\hline
\end{tabular}


Os dados provenientes do preenchimento da planilha mensal considerados para ilustrar este trabalho referem-se à soma da produção de todos os psicólogos residentes no período de fevereiro de 2006 a novembro de 2006, com exceção de dois meses, nos quais não foram realizadas atividades nas UBSs. 0 quadro a seguir mostra o número de pessoas atendidas por faixa etária em cada grupo de atividade, referente ao preenchimento da Tabela 1 da planilha e o número total de ações realizadas por grupo de atividades, referente ao preenchimento da Tabela 2 da planilha, o restante da tabela não será considerado para esta análise.

\section{Quadro 3 - Número de pessoas atendidas pelos residentes por atividade e ciclo de vida}

\begin{tabular}{|c|c|c|c|c|c|c|c|c|c|c|c|c|c|}
\hline & & & & & \multicolumn{4}{|c|}{ Grupo } & & & & & \\
\hline & \multicolumn{2}{|c|}{ Consultas } & \multicolumn{2}{|c|}{ Terapia } & \multicolumn{2}{|c|}{ Terapêutico } & \multicolumn{2}{|c|}{ Educativo } & \multicolumn{2}{|c|}{$\begin{array}{c}\text { Oficina } \\
\text { terapeutica }\end{array}$} & \multicolumn{2}{|c|}{$\begin{array}{c}\text { Visita } \\
\text { domiciliar }\end{array}$} & \multirow{2}{*}{$\begin{array}{r}\begin{array}{r}\text { Total por } \\
\text { faixa etária }\end{array} \\
n^{0}\end{array}$} \\
\hline & $n^{0}$ & $\%$ & $n^{0}$ & $\%$ & $n^{0}$ & $\%$ & $n^{0}$ & $\%$ & $n^{0}$ & $\%$ & $n^{0}$ & $\%$ & \\
\hline Crianças (de o aıı anos) & 171 & 34,5 & 343 & 28,2 & 983 & 43,8 & 2444 & 59,9 & 60 & 10,5 & 144 & 22,3 & 4145 \\
\hline Adolescentes (de 13 a 21 anos) & 77 & 15,5 & 224 & 18,4 & 132 & 5,9 & 259 & 6,4 & 63 & 11,05 & 102 & 15,8 & 857 \\
\hline Adultos (de 22 a 59 anos) & 237 & 47,8 & 630 & 51,9 & 1064 & 47,3 & 1179 & 28,9 & 433 & 76 & 336 & 52 & 3879 \\
\hline Idosos (6o anos ou mais) & 11 & 2,2 & 18 & 1,5 & 67 & 3 & 197 & 4,8 & 14 & 2,5 & 64 & 9,9 & 371 \\
\hline Total de pessoas atendidas & 496 & 5,4 & 1215 & 13,2 & 2246 & 24,3 & 4079 & 44,1 & 570 & 6,1 & 646 & 6,7 & 9252 \\
\hline Total de atividades & 477 & 18,5 & 1155 & 44,8 & 258 & 10 & 153 & 5,9 & 59 & 2,3 & 479 & 18,5 & 2581 \\
\hline
\end{tabular}

A partir do Quadro 3, constatou-se que os psicólogos residentes realizaram 2581 atividades que beneficiaram 9252 pessoas em regiões diferentes da Zona Leste de São Paulo. Verificou-se que um número maior de pessoas $(75,52 \%)$ foi beneficiado por atividades grupais (grupos: terapêuticos e educativos e oficinas terapêuticas), enquanto $25,74 \%$ foram atendidos em consultas, terapias ou visitas domiciliares, individualmente ou não. Os resultados mostraram que em todas as atividades realizadas, o número de atendimento foi maior de crianças ( $45 \%$ ) e adultos ( $42 \%)$.

\section{Reflexões, Análise e Contribuições}

A sistematização das atividades realizadas e a construção da planilha mensal não tinham como pretensão normatizar as ações do psicólogo no PSF, já que os modos de atuação devem ser elaborados de acordo com a realidade e as necessidades locais. Entretanto, a partir da reflexão, possível nas reuniões de supervisão, percebeu-se pontos em comum nas diversas intervenções realizadas pelos psicólogos residentes nas diferentes localidades.

Supõe-se que a configuração exposta neste trabalho seja enviesada por três aspectos principais: as diretrizes e pressupostos do PSF, a formação em psicologia e, por fim, a vinculação a um programa de formação multiprofissional em serviço.

Sobre as diretrizes do PSF, destaca-se que aspectos como a territorialidade, a intersetorialidade e a ênfase em ações de promoção da saúde e prevenção permearam as escolhas de ações educativas e grupais na unidade e na comunidade e ainda em outros setores como creches, escolas, associações de bairro, etc.

Em relação à formação em psicologia, segundo Dimenstein (1998), é possível apontar uma inadequação da formação profissional para a realização da psicologia nas Unidades Básicas de Saúde, já que o enfoque dos cursos de graduação é o clínico tradicional. Essa reflexão traz uma possibilidade de entendimento da escolha desses residentes por atendimentos clínicos na atenção básica, sendo típicos do nível médio e ambulatorial de atenção.

A consolidação da Psicologia como uma profissão de saúde pública (...) ainda não repercutiu significativamente na cultura profissional e leiga que permeia a atuação do psicólogo, já que as mudanças nos padrões de atuação ainda não são consistentes o suficiente para mudar as feições da prática psicológica no Brasil. (Oliveira, 2005, p. 282). 
Por fim, estar vinculado a um Programa Multiprofissional de formação em serviço influenciou a prática dos psicólogos residentes em dois aspectos principais. Inicialmente, ao permitir aos profissionais contar com a possibilidade de desenvolver práticas interdisciplinares e posteriormente, por não impor aos psicólogos residentes um compromisso com a demanda, mas sim com a formação e o aprendizado, o que evidencia aspectos como a possibilidade de experimentação e certa autonomia na realização das ações em saúde.

Cabe pensar que os psicólogos da rede do Sistema Único de Saúde poderiam ou não desenvolver atividades como as descritas neste trabalho, entretanto, para esses profissionais, existiria a influência de outros vieses; um dele possivelmente é o SIA-SUS, já que se propõe a ser um instrumento de codificação das intervenções feitas pelos profissionais, além de garantir o financiamento que mantém o trabalho, pois "as estatísticas computadas no SIA-SUS são responsáveis pela manutenção de programas, de financiamentos, de gratificações para os profissionais" (Oliveira, 2005, p. 278), o que significa que, ao planejar e propor ações em saúde, os psicólogos da rede são direcionados por esse Sistema de Informação.

Utilizou-se o SIA-SUS com a expectativa de se fazer uma intersecção entre a vivência dos residentes e o que é padronizado no SUS referente às possibilidades de atuação do psicólogo. 0 instrumento construído é o produto dessa tentativa de diálogo e serve de base para esta análise. Avalia-se, assim, que este estudo de caso, reforça a necessidade de adaptação ou construção de instrumentos que mais se aproximem dessa prática diferenciada, possível de ser realizada no PSF, para que ações de promoção realizadas pelos psicólogos na atenção básica possam ser visualizadas, planejadas, avaliadas e reconhecidas pelo sistema. Tornase urgente a análise crítica sobre a adequação dos códigos do SIA-SUS, sobre o enfoque e sobre o paradigma do conhecimento sob o qual ele é desenvolvido.

Ao utilizar um instrumento de sistematização quantitativa, foi possível visualizar informações como, faixa etária e quantidade de pessoas atendidas, carga horária e tipos de ações, e, conseqüentemente, surgiu a possibilidade de organizar, refletir e avaliar a abrangência e a adequação das práticas ao que é preconizado no PSF, bem como novas possibilidades de atuação.
Avalia-se que a sistematização numérica é fundamental para o acompanhamento e o planejamento das ações realizadas no PSF. Apesar desta relevância da quantificação, acredita-se que não se deva torná-la o cerne da atuação do psicólogo, tendo em vista, que a preocupação em atingir as metas esperadas, pode negligenciar as reais necessidades da população.

Dessa forma, de acordo com Oliveira e col. (2005), os números não permitem visualizar a quantidade de agravos que não ocorreram, ou seja, que foram prevenidos com as ações de educação e prevenção, embora a análise dos dados quantitativos seja responsável para a manutenção de programas de saúde. Portanto, reforça-se a importância da avaliação qualitativa da assistência prestada à saúde nos diversos níveis de atenção, principalmente no PSF.

Como afirma Dimenstein (1998), ainda inexistem pesquisas nacionais e locais que apresentem de forma sistemática a atuação do psicólogo nas UBS, o que dificulta a discussão acerca da atuação nesse campo específico.

Considera-se que este estudo de caso traz diversas contribuições para a formação de uma proposta coerente, sólida e comprometida com os ideais da reforma sanitária, para inserção do psicólogo no PSF, realidade possível com a implantação do NASF. Pelo Programa Multiprofissional de formação em serviço, surge a possibilidade de apropriação dos pressupostos e diretrizes do PSF, do reconhecimento das possibilidades de atuação do psicólogo neste contexto e da proposição de um instrumento de quantificação. Apresenta-se uma possível adaptação desse instrumento ao que é preconizado pelo Sistema de Informação Ambulatorial, entretanto, acredita-se que o grande desafio é a reformulação do SIA visando as a uma avaliação qualitativa que enriqueça a reflexão sobre a abrangência, a eficácia e a efetividade das ações em atenção básica do psicólogo.

\section{Referências}

ALVES SOBRINHO, E. J. M.; CAPUCCI, P. F. Saúde em São Paulo: aspectos da implantação do SUS no período de 2001-2002. Estudos Avançados, São Paulo, v. 17 , n. 48 , p. 209-227, 2003. 
BOURGET, M. M. M. et al. Residência

multiprofissional em saúde da família: a experiência da Faculdade e Casa de Saúde Santa Marcelina. In: BRASIL. Ministério da Saúde. Secretaria de Gestão do Trabalho e Educação na Saúde. Departamento de Gestão da Educação na Saúde. Residência multiprofissional em saúde: experiências, avanços e desafios. Brasília, DF, 2006. p. 109-122.

BRASIL. Constituição Federal, Código Penal, Código de processo penal. 8. ed. São Paulo: Ed. Revista dos Tribunais, 2006a.

BRASIL. Ministério da Saúde. Secretaria de Atenção à Saúde. Saúde mental e atenção básica: o vínculo e o diálogo necessários. Disponível em: <http:// portal.saude.gov.br/portal/arquivos/pdf/ diretrizes.pdf>. Acesso em: 7 dez. 20o6b.

BRASIL. Ministério da Saúde. Departamento de Atenção Básica. Atenção básica e a saúde da família. Disponível em: <http://dtr2004.saude.gov.br/dab/ atencaobasica.php>. Acesso em:19 jan. 2007a.

BRASIL. Ministério da Saúde. Portaria interministerial 2118. Disponível em: <http:// dtr2001.saude.gov.br/sas/PORTARIAS/Port2003/GM/ GM-2118.htm>. Acesso em: 23 jan. 2007b.

BRASIL. Ministério da Saúde. Sistema de informação ambulatorial do sistema único de saúde (SIA/SUS). Disponível em: <http://dtr2oo1.saude.gov.br/sas/ Decas/tabelasia.sih.htm>. Acesso em: 23 jan. 2007c.

BRASIL. Ministério da Saúde. Departamento de Atenção Básica. Propostas dos NASF é aprovada na Tripartite. Brasília, DF, 2007. Disponível em: <http:// dtr2004.saude.gov.br/dab/noticia/ noticia_ret_detalhe.php?cod=372>. Acesso em: 8 out. $2007 d$.
CAMARGO-BORGES, C.; CARDOSO, C. L. A psicologia e a estratégia saúde da família: compondo saberes e fazeres. Psicologia \& Sociedade, Ribeirão Preto, v. 17, n. 2, p. 26-32, maio/ago. 2005.

CAPISTRANO FILHO, D. O programa de saúde da família em São Paulo. Estudos Avançados, São Paulo, v. 13 , n. 35, p. 89-100, 1999.

CRP - CONSELHO REGIONAL DE PSICOLOGIA DE SÃO PAULO. Saúde mental no PSF é um desafio para a psicologia. Jornal PSI, São Paulo, n. 149, p. 6-7, out./ dez. 2006.

DIMENSTEIN, M. D. B. O psicólogo nas Unidades Básicas de Saúde: desafio para a formação e atuação profissionais. Estudos de Psicologia, Natal, v. 3, n. 1, p. 53-81, jan./jun. 1998.

OLIVEIRA, I. F. et al. A Psicologia, o Sistema Único de Saúde e o Sistema de Informações Ambulatoriais: inovações, propostas e desvirtuamentos. Interação em Psicologia, Curitiba, v. 9, n .2, p. 273-283, jul./dez. 2005 .

SÃO PAULO. Secretaria Municipal de Saúde. Documento norteador. Disponível em: $<$ http:// www.saudeprev.com.br/psf/saopaulo/pdf-geral/ DocumentoNorteador-Abril2005.php $>$. Acesso em: 18 jan. 2007a.

SÃO PAULO. Secretaria Municipal de Saúde. Documento norteador. Disponível em: <http:// www.prefeitura.sp.gov.br/downloads/ cartilha2.pdf(2002)>. Acesso em: 18 jan. 2007b.

VIANA, A. L. da A. Modelos de atenção básica nos grandes municípios paulistas: efetividade, eficácia, sustentabilidade e governabilidade. Ciência e Saúde Coletiva, Rio de Janeiro, v. 11, n. 3, p. 577-6o6, jul./set. 2006. 